\section{Conclusion.}

Reprenons le schéma de la figure $1 / 4$ ct supposons un défaut entre deux phases au point $P$.

Le schéma équivalent des impédances inverses comporte les mêmes branches que celui des impédances directes figure 15 ; seules les valeurs des impédances sont différentes.

Le courant de défaut sera :

$$
\text { I défaut }=\frac{U V \bar{Z}}{Z_{i l}^{\mathrm{t}}+Z_{i}^{\mathrm{t}}}
$$

$Z^{\mathrm{t}}$ désignant l'impédance totale résultante, directe et inverse.

\section{Remarque.}

Une approximation parfois acceptée pour la simplicité des calculs consiste à identifier le schéma des impédances transitoires directes à celui des impédances inverses.

$5^{\circ}$ Court-circuit alimenté par deux alternateurs (fig. 16).

Le schéma est identique au précédent.

On généralisera, comme il a été fait pour les impédances directes, au cas d'un réseau non bouclé constitué par un nombre quelconque de générateurs et de récepteurs.

II. - Impédances inverses des ḱléments d'un réseau.

Alternateur : l'impédance inverse a été indiquée ci-dessus.

Transformateur, ligne, récepteur inerte : l'impédance inverse est identique à l'impédance directe car elle est impédente et l'ondre cyclique des phases.

III. - Réduction des mpédances.

Le calcul se fera comme pour les impédances directes.

\section{IV. - Application numb́rigue.}

Court-circuit entre 2 phases d'un alternateur à vide :
En cas de court-circuit franc aux bornes

$Z_{e}^{a}=0 \quad Z_{c}^{i}=0$.

Pour un turbo-alternateur on aura en désignant par $I_{c c}$ tri le courant de c.c. entre les 3 phases et par $I_{c c}$ ph le courant de c.c. entre deux phases :

\begin{tabular}{|c|c|c|c|}
\hline$t$ & $Z_{d}$ & $Z_{t}$ & rapport $\frac{I_{c c} t r i}{I_{c c} \mathrm{ph} .}$ \\
\hline 0 & 12 & 12 & $\frac{24}{12 V \overline{3}}=1,15$ \\
0,1 & 19 & 12 & $\frac{31}{19 V \overline{3}}=0,95$ \\
permanent & 220 & 12 & $\frac{232}{220 V \overline{3}}=0,01$ \\
\hline
\end{tabular}

Le courant permanent de c.c. entre phases est donc 1,04 fois plus grand que le courant de c.c. triphasé.

Supposons maintenant une grande réactance extérieure, égale à 100; les valeurs directes et inverses de cette réactance étant égales.

\begin{tabular}{|c|c|c|c|}
\hline$t$ & $Z_{d}+Z_{c}^{i}$ & $Z_{i}+Z_{c}^{i}$ & rapport $\frac{I_{c c} \text { tri }}{I_{c c} \text { ph. }}$ \\
\hline 0 & 112 & 119 & $\frac{224}{112 \sqrt{3}}=1,15$ \\
0,1 & 119 & 112 & $\frac{931}{119 V \overline{3}}=1,12$ \\
permanent & 320 & 112 & $\frac{432}{390 / \overline{3}}=0,78$ \\
\hline
\end{tabular}

(à suivre)

\title{
La vie et les travaux d'André-Marie AMPÈRE
}

(1775-1836)

"Un physicien mathématicien de génie "

par V. Sruvestre, Ingénieur A.M. et I.E.G.

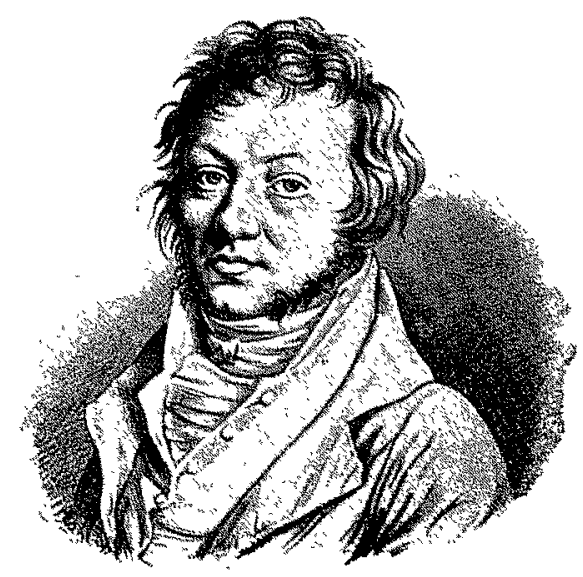

"Il était aussi grand Aussi simple, Qu'il était bon ».
Le 24 novembre 1793 Jean-Jacques Ampère, bourgeois de Lyon, ancien négociant en soie et Juge de paix du canton de la Halle aux blés, mîlé aux luttes politiques qui, en 1793, mirent à lyon aux prises girondins et jacobins, montait sur l'échafaud !

Dans une lettre que ce simple bourgeois adressait à sa femme la veille de mourir, il lui disait:

"Quant à mon fils il n'y a rien que je n'attende de lui "; et plus loin, au milieu de quelques notes d'économie domestique :

"Ma plus forte dépense a été lachat de livres et instruments de géométrie dont nótre fils ne pouvvait se passer pour son instruction, mais cette dépense était une sage économie puisqu'il n'a jamais eu d'autre maître que lui-même.

Ce fils était : André-Marie Ampère.

Né à Lyon le 22 janvier 1775 sur la paroisse Saint-Nizier, 
Ampère fut élevé dans l'humble village de Poleymieux situé à quelques lieues de Lyon, au milieu de ces doux coteaux du Mont-d'Or, où son père s'était retiré avec une modeste aisance.

C'est à Polcymieux qu'il passa la plus grande partic de sa jeunesse, sans autre maître que la nature, dans le oalme de la campagne et la méditation.

De bonne heure il manifesta des dispositions remarquables. A onze ans le jeune Ampère avait déjà étudié l'algèbre et la géométrie; les livres manquant dans la modeste bibliothèque de Poleymieux, on se rendit à Lyon, emprunter les ouvrages d'Euler et de Bernouilli ; mais pour pouvoir les lire, Ampère dut lestement apprendre le latin.

A dix-huit ans, il étudiait la mécanique analytique de Lagrange dont il avait, dit Sointe Beuve, refait tous les calculs ; et Ampère a répété depuis "qu'il savait autanl de malhématiques qu'il en a jamais su ".

Ce qui ne l'empêchait pas de lire dans le texte les auteurs latins et d'être indifféremment intéressé par : l'histoire, la botanique, la philosophie, l'histoire naturelle et aussi la poésie.

De celte poésie dont Ampère avait gardé toute sa vie un certain culte, nous donnerons l'élégie où il célèbre l'amour de Julie. Ce poème intitulé :

"Sur l'absence de l'adorée " a été lu par une jeune fille sous les tilleuls de la maison de Poleymicux lors de son inauguration, en juin 1928 :

"Que j'aime à m'égarer dans ces routes fleuries, Où je t'ai vue errer sous un dais de lilas :

Que j'aime à répéter aux nymphes attendries, Sur l'hesbe où tu t'assis, les vers que tu chantas !

"Au bord de ce ruisseau dont les ondes chéries Ont, à mes yeux, séduits, réfléchi tes appas, Sur les débris des fleurs que tes mains ont cueillies, Que j'aime à respirer l'air que tu respiras !

"Les voilà ces jasmins dont je t'avais parée, Cie bouquet de troenne a touché tes cheveux. Tout ici me retrace une image adorée.

Toul y plaint les tourments d'un amant malheureux.

"Regarde cette rose aujourd'hui desséchée ! Hier, elle exhalait les plus douces odeurs, Sur ton sein palpitant tu l'avais attachée! Quel injuste dédrain a flétri ses couleurs !

"Tout passe I C'est ainsi que la course des âges Sur les ailes du temps emporte nos beaux jours, Qu'un ciel pur et serein se couvre de nuages, Que l'absence succède aux plus tendres amours!

"O Fanny I C'est ici que mon âme éperdue Nourrira les chagrins dont je suis déchiré. J'y dirai tous les jours : "C'est lì que je l'ai vue ! En me disant adjeu c'est là qu'elle a pleuré l..."

Et, ces quelques ver's où Ampère célèbre Julie sous forme de chanson :
"Des cheveux d'or, des ycux d'azur', Un teint où l'on croit voir des roses Nager dans le lait le plus pur ; Sur les lèvres à demi closes D'une bouche digne des dicux, Un sourire naïf et tendre ; Une voix, pour être amoureux, Qu'il suffit seulement d'entendre ".

La mèche des cheveux de Julie trouvée dans les manuscrits d'Ampère montre bien que l'expression "des "chevenx d'or" devait ètre entendu dans son sens lilléral. Quant à la beaulé de Julie, peut-être avait-elle, comme l'a fait remarquer M. de Launay, ce charme si fréquent chez les femmes prédisposées à lit phtisie.

Entre la poésie el les mathématiques, Ampère absorbe la volumineuse encyclopédie de Diderot et d'Alembert, dont il pouvait, cinquante ans plus tard, grîce à sa prodigieuse mémoire, réciter des tirades entières.

A Lyon, où Ampère donne des leçons particulières de physique et de mathématiques, il se lie d'amilié avec un groupe de jeunes gens, les : Bonjour, Lenoir, Roux, Bredin et Ballanche ; ce Ballanche qui deviendra l'ombre fidèle de Mme Récamier, le philosophe que Chateatubriand appellera : Io mandarin ès lettres, le grand Ballanche !

On travaille ferme dans la petile chambre de la place des Cordeliers ; on discute : philosophie, chimie, mathénatique, religion, histoire et aussi métaphysique. C'est là qu'Ampère commente à ses amis l'ouvrage de Lavoisicr : Traité de Chymie d'après les découvertes modernes ", dont la doctrine nouvelle opérait comme une révolution dans la chimie.

Le 6 août 1799, Ampère épousa Mlle Julie Carron, du village de Saint-Germain, voisin de Poleymieux, et réalisait les joies rêvées et attendues avec une constance rare. Ils s'installent à Lyon et en août 1800, Julie Carron lui donne un fils appelé Jean-Jacques (1). Ce fils fera la joie et aussi le tourment d'Ampère, et circonstance peul-être unique, le père et le fils se trouveront plus tard tous denx professeurs au Collège de France!

En 1801, Ampère est nommé professeur de physique et chimie à l'Ecole Centrale de l'Ain, à Bourg. Sa fẹmme manifestant déjà le mal qui va la conduire bientôt à ja tombe, ne, peut l'accompagner et il part seul occuper son premier poste de professeur, emportant dans le cceur l'espoir de revenir à Lyon comme professeur au Lycée!

De Bourg, Ampère entretient avec sa femme une volumineuse correspondance sous forme de journal, où l'on trouve une fraîcheur de pensée el d'expressions vraiment peu communes. Au jour le jour, il rend compte à Julie de ses plus minutieuses affaires, ses labeurs, ses joies, ses désespoirs, ses travaux, el tout cela avec une candeur juvénile.

Son premier travail particulier est un mémoire sur la

(1) Jean-Jacques Ampère (1800-1864) avait hérité de l'intel(1) Jean-Jacques Ampere (18 ecrivit une tragédie. Introduit par allanche auprès de Mme Récamier et de Chateaubriand, voallanche aupres de ctudie les langues étrangères. Professeur de littérature n A thénée de Marseille, de littérature étrangère à l'Ecole normale A thénée de Marseile, des Lettres de Paris. En 1883 a l'âge de 32 ans, proesseur de littérature française au College de France. Membre de 'Académie des Inscriptions et Belles Lettres. Auteur d'une hisoire de la formation de la langue française, et de nombreux uvrages d'érudition. 
"Thérie malhémalique du jeu", qu'il envoie à Julic avec son fameux journal el ses effets, par les soins du messager Pochon. Celui-ci faisait douze lieues, de Bourg à Lyon, en dix heures, quand il ne restait pas en chemin, embourbé sur la route an milieu de l'hiver.

Ampère travaille beaucoup les mathématiques et il présente à la Société d'Emulation de Bourg un mémoire sur : "l'Application des formules des variations à la mécanique"; ce mémoire cst remarqué par M. Delambre, qui recommande Ampère el le fait nommer professeur de mathématique au Lycée de Lyon en 1803.

Ilćlas ! Ampère jouissait à peine de se trouver réuni à tout ce qu'il aimail que sa femme s'éteignait le 13 juillet 1803 . Aballu el désorienté, al ébauche des projets impossibles; cette place de professeur au Lycée de Lyon, il n'a plus le courage de loccuper, copendant que vers octobre 1804, sous la recommandation de M. Delambre, il est nommé : Répétiteur d'Analyse Mathématique à l'Ecole Polytechnique.

II quitte Lyon pour Paris où une nouvelle vie va commencer pour lui. Il se lie bientôt avec les métaphysiciens : de Tracy et Maine de Biran, ce qui fait dire à son ami Ballanche: "Songez bien qu'il n'y a que de très grands suceès qui puissent justifier votre abandon des mathématiques, où ceux que vous avez déjà cu, présagent ceux que vous devez attendre. Mais je sais que vous ne pouvez mettre un frein à votre cerveau !"

De 1805 à 1820, Ampère va pourtant faire marcher de front les mathématiques, la physique, la chimie, la mécanique, la physiologie, sautant d'un sujet à l'autre avec facilité el apportant dans toutes ces branches des sciences des idées nouvelles.

Il devia encore suffire aux différents emplois dont il va être successivement surchargé :

Membre du Bureau Consultatif des Arts et Méticrs,

Inspecteur Général de l'Université en 1808,

Professeur de Philosophie à la Faculté des Lettres en 1819,

Professeur suppléant d'Astronomie à la Faculté des Sciencosen 1820 .

En 1818, Ampère s'installe dans une petite maison achetée avec sa sœur, nue des Fossés-St-Victor (aujourd'hui rue du Cardinal. Lemoine).

C'est dans cette maison qu'Ampère s'était réservé une pièce, où il avait équipé à ses frais un modeste laboratoire muni d'instruments de fortune avec lesquels il mènera à bien pourlant toutes ses recherches et expériences sur l'électromagnétisme et l'électrodynamique.

Et voici qu'arrive le moment où va en quelque sorte éclater le génie d'Ampère! Vers septembre de l'année 1820, arrive a Paris par la Suisse la nouvelle de la découverte du physicien danois Oersted, lequel avait constaté que :

"l'aiguille aimantée est déviée lorsqu'elle est placée au "voisinage d"un fil métallique parcouru par un courant".

Le 11 septembre 1820, Arago répète devant l'Académie l'expérience d'Oersted. Ampère est immédiatement frappé par ce phénomène, auquel il avait sans doute déjà songé lorsqu'il était à Bourg IIl paraît en saisir déjà toutes les conséquences.

Il se met immédiatement au travail dans son cabinet de la rue des Fossés-Saint-Victor, et sept jours après il présente devant l'Académie des Sciences son mémoire :
"Nouveaux phénomènes gaivanico-magnéliques et explications des expériences d'Oersted", et y refait les expériences en donnant tous les commentaires.

Le même jour, il écrit à son fils, qui royage en Suisse : "Tous mes moments ont été pris par une circonstance importante de ma vie depuis que j'ai entendu parler pour la première fois de la belle découverte d'Oersted, professeur à Copenhague, sur l'action des courants galvaniques sur l'aiguille aimantée.

"J'y ai pensé constamment, je n’ai fait qu'écrire une grande théorie sur ce phénomène et tous ceux déjà connus de l'aimant et tenté des expériences indiquées par cette théorie, qui toutes ont réussi et m'ont fait connaitre autant de faits nouvcaux. Voilà une nouvelle théorie de l'aimant qui en ramène par le fail tous les phénomènes à ceax da galvanique. Cela ne ressemble en rien à ce qu'on en disait jusqu'à présent." "

En quelques semaines, Ampère remet à l'Académie toute une série de mémoires sur l'uclion des courants entre eux, des aimants et des courant.s et anssi sur l'action magnétique de la terre sur l'aiguille aimantée.

Ampère parvint à réaliser toutes ces expériences de la façon la plus ingénieuse au moyen d'une série d'appareils extrêmement simples montés sur une table restée classique sous le nom de "Table d'Ampère ". C'est cette fameuse table qu'il avait dans son cabinet de la rue des Fossés-Saint-Victor et qui, pieusement conservée au Collège de France, est venue, ces dernières années, enrichir le Musée Ampère à Poleymieux.

Ampère possédait un instrument précieux dont il jouait à la perfection depuis sa jeunesse, celui de l'analyse mathématique et qui lui permit de relier les faits entre eux et remonter aux sources, pour enfin formuler les lois dont l'extrême simplicité contraste singulièrement avec la complexité au premier abord inextricable des phénomènes à étudier.

Tous ces travaux se trouvent résumés dans la "Théorie des phénomènes électrodynamiques uniquement déduite de l'expérience " publiée en 1826 ; œuvre admirable que l'illustre physicien anglais "Maxwell " déclarait, il y a déjà plus de cinquante ans, être un des plus grands exploits de la science.

Ampère voit défiler dans son cabinet de la rue des FossésSaint-Victor de nombreux savants français et surtout étrangers, et avec la meilleure grâce du monde il leur répète ses expériences. Mais, comme dans toute théorie nouvelle qui bouleverse peu ou prou les idées et aussi les réputations acquises, les objections s'élèvent ; les plus acharnées proviennent de cartains de ses collègues de l'Académie.

Ampère répond à tout et à tous avec une courtoisie, une bonté digne d'une grande âme.

C'est qu'il y avait chez lui un admirable désintéressement. soientifique quui lui faisait désirer le progrès de la vérité pour la vérité même, sans aucune considération de gloire et de profit personnel.

En 1824, il est nommé professeur de physique générale et expérimentale au collège de France, ce qui ne l'empêche pas à cette époque, de publier des études sur la théorie atomique, sur les lois de la réfraction avec Fresnel et un travail curieux d'anatomie comparée sur les animaux articulés.

Ampère, en prouvant l'identité de l'électricité et du magnétisme, avait réalisé l'œuvre qui mettait le sceau à sa gloire 
scientifique, mais comme nous l'avons déjà vu, son esprit élait universel, a la plus grande joie de ses auditeurs au collège de France.

Sa grande préoccupation arait été la philosophie et la classification des sciences; il arait mème commencé l'élaboration d'une langue universelle!

Vers 1830, son fils disait :

"Les grands problèmes sociaux qui avaient loujours préoccupé mon père faisaient partie pour lui d'une science qu'il appelait : la science de la félicité publique $n$.

A l'époque où Ampère allait rétablir sa santé dans le Midi, vers l'année 1828 , et que, voyageant en diligence en compagnie de son fils et d'un ami, c'était des causeries interminables sur les sujets les plus élevés de la philosophie ef de l'histoire, comme il en avait seul le secret et la facililé. Comme son fils lui reprochait un jour de trop se fatiguer, il répondit :

"Ma santé ! ma santé ! il s'agit bien de ma santé. Il ne doit ètre question entre nous que des vérités éternelles, des choses et des hommes qui ont été ntiles on funcstes à l'humanité ".

Voilà quel était l'homme! profondément bon et humain.

Dans la préface que son fils avait préparée en 1856 à l'introduction à la Philosophie d'André-Marie Ampère, il est dit : "Chez mon père les sentiments de l'homme étaient d'accord avec la doctrine du psychologiste. La timidité de ses manières, suite d'une jeunesse passée dans ses montagnes de Poleymieux, ne l'empêchait pas d'aimer la liberté avec ardeur. André-Marie Ampère, pen habile à diriger sa fortune, toujours généreux avec de plus pauvres que lui, avait donné beaucoup aux siens et à part le traitement de ses places, il ne possédait presque rien.

Au cours d'une tournée d'Inspection des Ecoles, en juin 1836, Ampère arrivait à Marseille extrêmement fatigué et du collège de cette ville où il était soigné, il envoie à son fils Jean-Jacques ce billet laconique :

"Je succombe à la fatigue d'écrire.

"Je te quitte :avec une tendresse que rien ne peut égaler".
Il meurt le 10 juin 1836 et est inhumé à Marseille. Sur sa pierre tumulaire son fils faisait graver ces mols: "Il fut aussi bon, aussi simple qu'il était grand".

Ce n'est qu'en 1869 que ses restes furent transporlés à Paris, on il repose depuis au cimetière Montmartre, à côlé de son fils Jean-Jacques, l'ami malhe'ureux de Mme Récamier.

C'est en mai 1021 que la tombe dos deux Ampère a été restaurée par les soins picux ef reconnaissants du Syndicat français des Eloctriciens.

En juillet de la même année, un monument à la gloire d'Ampère étail ćrigé à Poleymicux, puis en 1927 MM. Sosthène et Hernand Behn achelaient et faisaient don de la maison de Poleymieux à l'Académie des Sciences, maison restaurée et inaugurée en juin 1928 par le Groupe du Sud Est de la Société française des Electriciens.

Enfin en juillet 1931, le Musée Ampère était ouverl a Polermieux; ce musés, organisé et sous la garde de la Société dos Amis d'André-Maric Ampère, est d'une touchante simplicité dans sa grandeur scientifique.

Faisant allusion à la fin du grand sarant lyounais, Sairte Beuve disait :

"En reportant son regard du haut de la montagne de la vie rers ces sciences quil comprenait toutes et dont il avait agrandi l'une des plus belles il put alleindre un moment de bonheur serein du sage ret reconnaîle en souriant ses domaines. "

Mais, si Ampère revenait aujourd'hui reconnaître ses domaines, que verrait-il ?

D'immenses usines génératrices captant l'énergie emmagasinée dans les neiges éternelles el par les siècles dans la terre, actionnant d'énormes machines déversant l'énergie électrique sur de vasles réseaux distribuant la lumière et la force motrice du palais à l'humble chaumière, la matière inertc fondue transformée par des réactions invisibles, la voix et la pensée tnansmise instantanément par toute la teare $! .$. et comme l'a dit M. Boucherot : devant tant de magnificences, Ampèrc croirait les hommes réconciliés, son rève réalisé et son cœur déborderait d'amour et d'allégresse.

\title{
A propos des célèbres travaux d'André-Marie Ampère sur l'électrodynamique
}

\author{
Par Y. Syzyestre; Ingénieur A.M. et I.E G.
}

Tous ceux qui ont étudié les travaux d'André-Marie Ampère ont été frappés par l'étonnante rapidité avec laquelle il a tiré toutes les conséquences de la loi d'Oersted et réalisé ses célèbres expériences sur l'électrodynamique.

On se rappelle en effet, que c'est le 11 septembre 1820, qu'Arago répète deviant l'Académie des Sciences l'expérience du physicien danois Oersted constant que :

"l'aiguille aimantée est déviée lorsqu'elle est placée dans le voisinage d'un fil métallique parcouru par un courant".

Ampère se trouve comme illuminé par cette simple expérience et parait en saisir instantanément toutes les conséquences; il voit notamment que :

"le voisinage d'un courant est un champ de force"
Il se met au travail dans son cabinet de la rue des FossésSaint-Victor, et au bout d'une semaine, le 18 septembre, il remet son premicr mémoire relatif.

"Au nouveau phénomène galvanico-magnétique et explicalions des expériences d'Oersled ", suivi du 18 au 25 septembre d'une séric de sept mémoires.

A la date du 25 septembre 1820, il fait devant l'Académie des Sciences les expériences sur les :

"phénomènes galvanico-magnétiques " en donnant tous les commentaires. Ce même jour il écrit à son fils Jean-Jacques qui royage en Suisse, la lettre suivante :

"Tous mes moments ont été pris par une circonstance " importante de ma vie depuis que j'ai entendu parler pour 
" la premièc fois de la belle découverte d'Ocrsted, profes"scur à Copenhague, sur l'action des courants galvaniques "sur l'aiguille aimantée.

"J'y ai pensé continuellement, je n'ai fait qu'écrire une " grande théoric sur ces phénomènes el tous ceux déjà con" nus de l'aimant el lenté des expóriences indiquées par cette " théorie, qui toutes ont réussi et m'ont fait connaître au"lant de faits nouveaux.

" Je lus le commencement d'un mémoire à la séance de "lundi, il y a aujourd'hui huit jours. Je fis les jours sui" vants, tantôt avec Fresnel, tantôt avec Depretz, les expé" riences confirmatives. Je les répétais toutes vendredi chez "Poisson, où s'étaient réunis les deux Demussy, plusieurs "élèves de l'Ecole Normale, le général Campredon, etc...

"Tout réussit à merveille, mais l'expérience décisive que " j'avais concue comme preuve définitive exigeait deux pi"les galvaniques, tentée avec des piles trop faibles chez-moi " avec Fresncl, elle n'avait point réussi.

"Enfin hier, j'obtins de Dulong qu'il permit à Dumotier " de me vendre la grande pile qu'il faisait construire pour "le cours de physique de la Faculté et l'expérience a ćté fai" te chez Dumotier arec un plein succès et répétée aujour" d'hui à 4 heures à la séance de l'Institut.

"Voilà une nouvelle théorie de l'aimant qui en ramène par " le fait, tous les phénomènes à ceux du galvanique.

"Cela ne ressemble en rien à ce qu'on disait jusqu'à pré. " sent ".

Certains ont vu dans cet éclair de génie comme une sorte do divination heureuse ; mais quelle erreur, la méthode d'Ampère est le fruit d'unc étude sérieuse et de délibérations mûres d'un esprit de premier ordre de son époque.

Son ceuvre de mathématique seule, comme l'a dit M. Appel, serait de naturc à le placer au premier rang parmi les mathématiciens qui ont créé les mathématiques modernes cl l'égaler au plus illustre d'entr'eux.

Ampère s'est servi avec rare lalent des mathématiques comme instrumenl de mathématique physique ; toutes les déductions des lois de l'électromagnétique ont été faites par lui à l'aide d'équalions différentielles exprimant, comme l'a fail remarquer M. Mailloux, des rapports entre des parties infiniment petites de circuits électriques ou d'aimants.

La lucidité et la profondeur de ses vues provenaient de son esprit universel. En 1820 , Ampère avait 45 ans ! il était depuis longtemps entrainć aux spéculations de l'analyse mathémalique, de la physique et de la philosophie.

Maxwell déclarait, il y a déjà plus d'un demi siècle que l'œuvre d'Ampère était:

\section{"l'un des plus grands cxploits de la science".}

Que cet exploil ail été réalisé en l'espace de quelques jours nous a frappé à notre tour ! Nous avions toutefois l'impression que la question de l'électromagnétisme avait en quelque sorte couvée longuement dans son esprit et que la découverte d'Oersted n'a élé que l'élincelle qui a brusquement illuminé le cerveau génial d'Ampère.

L'évènement en effel paraît subil, parce que ses origines, sa génèse nous onl b́chappé, l'œuvre de génie n'en est pas moins l'aboutissement d'un long travail progressif et secret.

Ce qui parait justifier nolre impression, c'est ce que dit Anupère lui-même I Nous avons parcouru attentivement Ia correspondance qu'il echangeait de Bourg avec sa femme,
Tulio Carron, des l'année 1802 . Nous rappelons qu' cette époque, Ampère professait la physique d la chinic a l'Ecole Centrale de l'Ain, à Bourg et qu'il avail dû, pour des raisons de santé de sa femme et aussi d'écononie, la laisser avec son fils Jean-Jacques à Saint-Germain-au-Monl-d'Or', près Poleymieux.

Dans celle intéressante correspondance et dans le famcux journal d'Ampère, nous trouvons ceci :

Ampère à Julie.

"J'ai commencé un nouveau travail car c'est le moment "d'étudier quand je suis loin de toi. Ne me servirait-il ja"mais de rien, j'aurai toujours gagné les connaissances ac"quises le préparant $1 "$.

Et dans le post-scriptum de la lettre que Julie lui adresse en 1802, elle pose cette curieuse question à son mari :

"Que penses-tu de ce que dit Bonaparte pour le galvanisme?n.

Bonaparte en 1802 lançait l'appel suivant aux chercheurs : "Je désire donner un encouragement de 60.000 francs ì " celui qui par ses expériences et ses découvertes fera faire "à l'électricité el au galvanisme un pas comparable à celui " qu'ont fait faire à ces sciences Franklin et Volta ".

De Bourg, Ampère répond à Julie par ces mots caractéristiques:

G'est un prix de soixante mille francs proposé par Bona" parte que je tâcherai de gagner quand j"en aurai le temps. "C'est précisément le sujet que je traite dans l'ouvrage sur " la physique que j'ai commencé d'imprimer mais il faut le "perfectionner et confirmer ma théorie par de nouvelles ex"périences".

C'est net, Ampère avait donc songé dès l'année 1802 aux problèmes des courants électriques et des aimants, mais il n'avait eu à cette époque ni le temps ni les moyens matéricls de tenter des expériences.

En effet, à Bourg, Ampère avait, en dehors de son cours de physique et de chimie à l'Ecole Centrale de l'Ain et les loçons particulières qu'il donnait à la pension Dupras et Olirier, été surtout absorbé par des recherches mathématiques.

Il avait publié deux importants mémoires, le premier :

"Considérations sur la théorie mathématique du jeu" avait été présenté à l'Institut par Laplace, et l'avis unanime des membres de la section des mathématiques était que cet ouvrage ne pouvait venir que d'une tête forte;

le deuxième :

"Application à la mécanique des formules du calcul des variations ".

avait été lu par Ampère à la Société d'Emulation de Bourg, lors d'une séance organisée en l'honneur des inspecteurs MM. Delambre et Villars, et très remarqué par ceux-ci.

Au surplus, Ampère avait préparé en collaboration aveo un Monsicur Clerc, professeur de mathématique à Bourg, un ouvrage classique :

"Leçons élémentaires sur les séries et autres formules indéfinies $n$.

Cet ouvrage quoique très avancé ne se termina jamais. I! ne faut pas oublier que pendant son séjour à Bourg de 1801 à 1803 la grande préoccupation d'Ampère et le but qu'il poursuivait était d'arriver à oblenir une chaire de malhémalique an Lycée de Lyon, afin de se rapprocher de sa femme 
et de son fils qu'il avail laissés à St-Germain-au-Mont-d'Or, près de Poleymieux.

Son ouvrage sur la physique resta inachevé et peut-être le manuscrit fut il perdu sur la route de Bourg à Lyon par le fameux messager Pochon, à qui Ampère confiait le soin de transporter vers sa femme, son journal, ses manuscrits, ses maigres appointements et ses effets. Ce n'est que dix-huit ans plus tard, à l'annonce de la découverte d'Oersted (1820)
qu'Ampère reprenant son ouvrage commencé à Bourg sur la physique, mettra le sceau à sa gloire scientifique par ses ra-. pides et immortels travaux sur l'électrodynamique.

Nous serions particulièrement satisfaits que la remarque que nous avons faite d'après lc fameux journal d'Ampère, puisse apporter une lumière, si faible soit-elle à l'histoire des travaux de l'illustre savant lyonnais dont notre pays a célébré solennellement le centenaire.

\section{DOCUMENTATION}

\section{ACADEMIE DES SCIENCES}

Séance du 2 Décembre 1935

PHYSIQUE THEORIQUE. - Sanliago Antunez de MAYOLO (prés. par M. Louis de BROGLIE). - Interprétation du coefficient de structure fine.

Pour interprêter le coefficient $\alpha=2 \pi e^{2} / \mathrm{h}$ c de structure fine, l'auteur se sert de la relation remarquable

et arrive à l'expression ;

$$
\alpha=\frac{2 \pi l^{2}}{h c}=\frac{l^{2}}{\varphi^{2}}
$$

a est le quotient de deux charges universelles notables : l'une $l$ différentiée propre de l'électron corpusculaire, et l'autro $Q$ non différentiée de tournure mathématique, caractéristique du champ électromignétique et liée au quantum $h$. On pourrait encore exprimer $a$ par le quotient $k / K$ du confficient $k$ de la loi de Coulamb appliquée aux corpuscules électriques dans l'atome de Bohr, et $\mathrm{K}$ propre du champ électromagnétique pour $\mathrm{M}=m_{\mathrm{o}}$.

\section{L'Électrification de la Ligne Vierzon-Brive}

Ce tronģon est le dernier en date qui ait été mis en service du vaste programme des grands travaux d'électrification français, c'est d'abord à ce titre qu'il nous semble utile de le menticnner, mais surtout ce sont les innovations qu'il comporte qui en font l'intérêt capital.

La longueur de cette nouvelle ligne électrifiée est de $300 \mathrm{Km}$. en double voie. L'intensitó très élevée du trafic de da région qu'elle dessert, la proximité des oentres producteurs d'énergie hydro-électrique du Massif Central, ainsi que son tracé relativement assez accidenté expliquent tout l'avantage dans l'exploitation qui doit résulter de sion électrification.

Le courant est distrilué aux lignes caténaires par l'intermédiaire de douze sous-stations, qui transforment le courant triphasé $90 \mathrm{Kv}$. 50 pér./sec. en courant continu à 1.500 v. Chacune d'elle comprend deux groupes de deux commutatrices en séric à $750 \mathrm{v}$. Lour fonctionnement est entièrement automatique.

Dans cefte très brève analyse, nous nous proposons de ne nous arrêter qu'aux particularités de conception qui nrésentent une cerlaine originalité par rapport aux installations des sousstations existantes sur le reste des réseaux électrifiés en France et dans cet esprit nous signalons la tentative faite dans trois sousstations, par l'adoption, à titre d'essai pour l'installation des barres à haute tension $(90 \mathrm{Kv})$ qui sont toujours en plein air, de la dispo. sition dite "barres à plat ". Celle-ci permet de réduire an minimum l'importance des charpentes métalliques el !e principe en est le suivant: au lieu d'être suspendus, les jeux de barres sont posés sur des isolateurs rigides, cux-mêmes supportés par des colonnes en béton.

D'autre part, du fail de la présence sur la ligne de nombrouses rampes de $10 \mathrm{~m} / \mathrm{m}$, Ies sous-stations qui se trouvent aménagées dans ces régions plus accidentées, ont été prévucs pour pouvoir. fonctionner en récupération.

Quant au matériel roulant, il nous semble à propos de signaler que la Compagnie d'Orléans a augmenté, en prévision de celte nouvelle ligne à exploiter. son important parc de locomotives (en grande partie des locomotives à grande vitesse du type 2 B 2 avec commande Büchli) d'un certain nombre de locomotives du mème type, mais au cours de l'année écouléc elle a mis en service trois types do machines nouvelles présentant des caractéristiques très particulières ef différentes, visant, d'unc part, à lamélioration des facilités de démarrage et des possibilités de remorques de trains lourds en terrain plat et en rampe de $10 \mathrm{~m} / \mathrm{m}$., el d'autre part, à la recherche de la simplicité et de la plus grande sécurité dans les dispositifs de commande.

Les conditions imposées sont : de remorquer, à la vitesse soutenue de $120 \mathrm{Km} / \mathrm{h}$ des rapides de $800 \mathrm{~T}$ sur palier, et de $751 \mathrm{~T}$ sur des parcours comportant de Jongues rampes de $10 \mathrm{~m} / \mathrm{m}$.

Parmi les types présentés, la 2R2, E 704, du type Westinghouse, construite par le groupement M.T.E. (Schneider, Matériel électrique S.W et F.A.C.E.I) a pu être réalisé en restant en dessous du poids imposé de 136.500 T. en ordre de marche, et cela malgré la présence de l'équipement spérial de récupération et sans avoir eu recours à l'utilisation de métaux légers.

La commande individuelle des quatre essieux moteurs est particôlièrement simple et robuste. Chaque essieu cst attaqué par un moteur cnlièrement suspendu fixé au châssis de la locomotive et entrainant par engrenages un arbre creux ou " quill " supportant un dispositif élastique à ressorts. Ce système de la Compagnio Westinghouse est encore amélioré en utilisant pour le guidage des coupelles élastiques, une armature monobloc présentant de grands avantages.

Toutefois, la carnctéristique la plus intéressante de celte machino réside daus le type de moteurs de traction don't les dispositions internes très particulières ont permis une réalisation allégée ot d'enmulement très réduit.

D'après les prévisions, les puissances-jantes pour l'ensemble à $1.500 \mathrm{~V}$. à l'a caténaire devaient êtro de $4.400^{\circ} \mathrm{CV}$. au régime unihoraire et $3.800 \mathrm{CV}$, au régime continu. En réalité, il a été constaté que la puissance disponible dans les conditions de définition (lension et échauffements) pouvaient être de $5.500 \mathrm{CV}$. en régime unihoraire et de $4.600 \mathrm{CV}$, en régime contir.u.

Co qui illustre le mieux les progrès attcints dans cette machine, c'est lo poids très réduil de celle-ci par cheval, qui ast de $24.700 \mathrm{Kg}$. nar cheval unihoraire et do $29.500 \mathrm{Kg}$. 1 mo cheval continu, en dépit de son équipement de récupération, el ccla sans avoir employé aucun métal léger.

Quant à l'équipement des sous-stations, nous ne signalerons que les particula rilés du schéma cmployé pour le couplage d'un' groupe de traction, celles-ci etant imposées par le fonctionnement entierement aufomatique des sous-stations. Cet équipement particulier 